\title{
Gefährliche Enge
}

\section{Fallbeispiel chronisch obstruktive Lungenerkrankung} „Organsystemübergreifendes, vernetztes Denken“ ist der Schlüssel zum physiotherapeutischen Dialog mit dem „Organismus Mensch“. Am Beispiel der chronisch obstruktiven Lungenerkrankung zeigt der Artikel ein strukturiertes Behandlungsvorgehen auf.
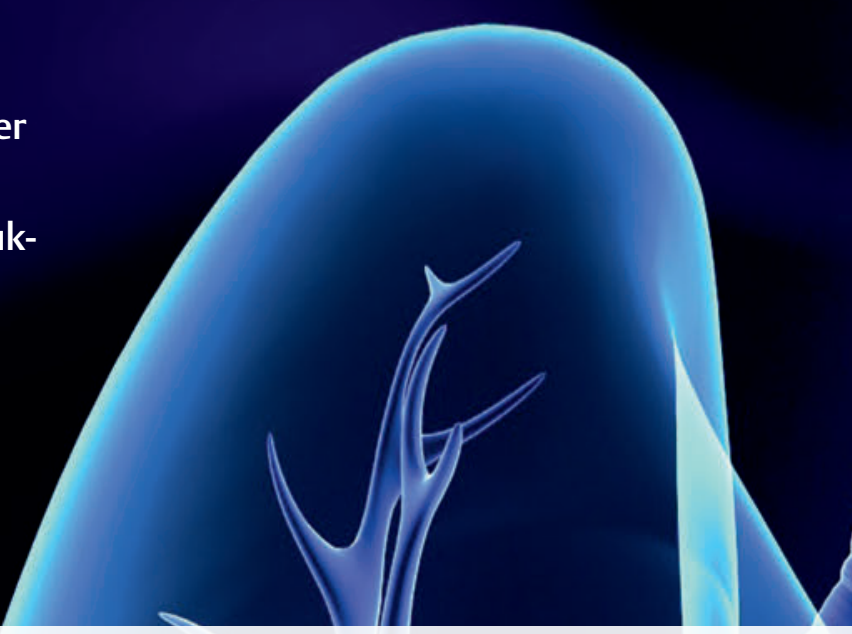

$\rightarrow$

Organsystemübergreifend und vernetzt denken - gerade in der Physiotherapie in der Inneren Medizin spielt das eine große Rolle. Dass hier viele Organsysteme ineinandergreifen, erkennt man beispielsweise am respiratorischen System. So sind die Lungen vor allem für die Sauerstoffaufnahme und die Kohlendioxidabgabe zuständig. Dieser Prozess erfolgt im Lungenparenchym, dem funktionellen Anteil der Lungen. Dort findet die „äußere Atmung“, der Gasaustausch, statt. Das HerzKreislauf-System ist für den Transport zum und vom Zielgewebe weg zuständig. Im Zielgewebe findet die „innere Atmung“ statt [1]. Ohne Grundkenntnisse dieser Körperstrukturen und -funktionen sowie der pathophysiologischen Veränderungen durch eine Erkrankung ist ein klinischer Denkprozess, ein Clinical Reasoning (CR), nicht möglich.

Möchten Therapierende nun die Situation eines Patienten oder einer Patientin mit einer Lungenerkrankung erfassen, braucht es Fakten. Doch wie viele Informationen benötigt man, um im CR-Prozess Hypothesen zu formulieren und diese danach zu bestätigen oder zu verwerfen?

Struktur- und Funktionshypothese $\rightarrow$ Markus Schenker differenziert in seinem Buch „Analytische Atemphysiotherapie“ zur Hypothesenformulierung in der Pneumologie die Strukturhypothese und die Funktionshypothese. Eine frische oder chronische Läsion einer Struktur (Strukturebene) hat Auswirkungen auf die Funktion (Funktionsebene). Ein operativer Eingriff am Thorax stört die strukturelle Integrität: Es treten Schmerzen bei Bewegung auf, die Atembewegungen sind meistens vermindert, es folgt eine Minderbelüftung. Auch eine chronische Vernarbung (Remodeling) der Atemwege bei sich wiederholenden Entzündungen ist eine strukturelle Veränderung. Eine Strukturhypothese beschreibt die Läsion einer oder mehrerer
Strukturen [2]. Die Verteilung der Luft in den Lungen, die Belüftung, der Gasaustausch und die Durchblutung gehören zu den Funktionen der Lunge. Eine Funktionshypothese beschreibt die Störung einer oder mehrerer dieser Funktionen [2].

"Lungenmobil Merz" als didaktische Hilfe $\rightarrow$ Eine didaktische Hilfe zur Berücksichtigung wichtiger Faktoren während der Beurteilung einer Patientensituation in der Pneumologie unter Einschluss der verschiedenen Ebenen der ICF ist das „Lungenmobil Merz“ (॰ ABB. 1).

Die Räder des Lungenmobils sind Parameter der klinischen Untersuchung. Der Einbezug all dieser Parameter unter Berücksichtigung der Kontextfaktoren erlaubt Therapierenden eine umfassende Beurteilung der klinischen Situation.

Die Spirometrie gibt Auskunft über die Leitfähigkeit der Atemwege und die Entfaltung der Lunge. Die Blutgasanalyse macht eine Aussage über den Gastransport und das Säure-Basen-Gleichgewicht. Diese Räder des Lungenmobils werden der Funktionsebene zugeordnet. Das Röntgenbild und die bildgebenden Verfahren stellen Strukturen dar. Sie geben einen Einblick in die Strukturebene. Die Klinik und das Verhalten in der Belastung ermöglichen eine Einschätzung der Belastbarkeit. Das Beachten der Achsen und Verbindungen des Lungenmobils schult die Vernetzung von Klinik, Strukturebene und Funktionsebene verschiedener Organsysteme. Es erlaubt den Übertrag auf die Aktivitäts- und Partizipationsebene. Die Achsen des Lungenmobils sind Verbindungen der Ebenen:

$\rightarrow$ Röntgenbild-Blutgaswerte-Spirometrie (Bezug Struktur Funktion)

$\rightarrow$ Röntgenbild-Klinik-Auskultation-Belastungstest (Bezug Struktur - klinisches Muster - Funktion)

$\rightarrow$ Spirometrie - Belastungstest (Lungenfunktion und Auswirkung auf die Belastbarkeit) 
$\rightarrow$ Spirometrie - Blutgaswerte (Lungenfunktion und Auswirkung auf den Gasaustausch)

Das Lenkrad hat eine Wechselbeziehung mit den Rädern. Die Problemanalyse als Lenkrad verbindet und vernetzt die verschiedenen Parameter miteinander. Als Ganzes vermittelt das Lungenmobil ein Bild des aktuellen Zustands von Patient*innen auf der Strukturund Funktionsebene sowie ein Bild der Belastbarkeit mit Berücksichtigung der Kontextfaktoren. In das Lungenmobil einzusteigen, verlangt eine Auseinandersetzung mit der Struktur und der Funktion sowie der Pathophysiologie der Lungen und des Herz-KreislaufSystems. Weitere durch Komorbidität bedingte Faktoren und Organsysteme werden auf der Fahrt integriert.

Fahren mit dem Lungenmobil braucht Übung durch Reflexion. Der Gewinn liegt im klaren Abstecken der therapeutisch sinnvollen Interventionen. Das Lungenmobil ermöglicht ein problemorientiertes Handeln sowie ein selbstkritisches Überprüfen der gewählten Maßnahmen. Das folgende Fallbeispiel zeigt dieses Vorgehen auf.

\section{Fallbeispiel}

Anamnese $\rightarrow$ Der 62-jährige Herr Müller, dessen Name redaktionell verändert wurde, leidet an COPD mit dem Schweregrad GOLD 2 (Global Initiative for Chronic Obstructive Lung Disease). Er kommt mit folgender Verordnung zur Physiotherapie: Verbesserung der Muskelfunktion und der kardiopulmonalen Funktion sowie Mukusmobilisation. Bei der ersten Begegnung verzieht Herr Müller das Gesicht und klagt über heftige, einstechende Schmerzen im rechten Thorax. Er sagt: „Das kommt von den vielen Hustenattacken.“ Der übergewichtige Patient hat Mühe, sein T-Shirt auszuziehen. Die Bewegung ist trotz der Einnahme von Schmerzmitteln für ihn sehr schmerzhaft. In den letzten Monaten war der 62-Jährige

\section{ஜ̊ Gewinnen}

Physiotherapie in der Inneren Medizin

Wer mehr über das Praxiswerk „Physiotherapie in der Inneren Medizin“ lesen möchte, kann eines von drei Exemplaren gewinnen. Im Buch geht es unter anderem um Clinical

Reasoning, Assessments und Training in der Inneren Medizin, Hilfsmittel und apparative Unterstützungsmethoden sowie physiound atemtherapeutische Techniken. Wer sich ein Buch sichern will, klickt bis zum 9.2.2022 unter www.thieme.de/physiopraxis > „Gewinnspiel“ auf das Stichwort „Innere Medizin“. Viel Glück!

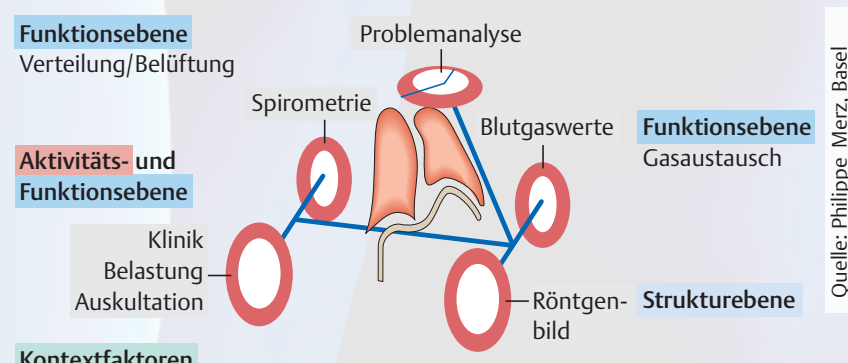

Kontextfaktoren

ABB. 1 Das „Lungenmobil Merz“ dient als didaktische Hilfe bei der Therapieplanung. 
oft mit seinem Motorrad unterwegs. Seinen Tag verbringt er überwiegend sitzend am PC. Die Nachtruhe sei wegen des Hustens und einer Schlafapnoe gestört. Er hat in Kürze einen Termin im Schlaflabor. Im Anschluss daran wird er mit großer Wahrscheinlichkeit eine Atemhilfe für die Nacht erhalten. Weiter erzählt der Patient, dass das Sekret nur schwer mobilisierbar sei, eine zähe Konsistenz besitze und dass das Abhusten ihn ermüde. Die Inhalation mit bronchienerweiternden Medikamenten zweimal am Tag schildert er als unproblematisch.

Befund $\rightarrow$ Herr Müllers Befund setzt sich aus folgenden diagnostischen Maßnahmen zusammen:

$\rightarrow$ Inspektion: In Ruhe ist das Atemmuster bis auf den einen oder anderen Aussetzer (der Patient vergisst zu atmen) unauffällig. Die periphere Sauerstoffsättigung $\left(\mathrm{SpO}_{2}\right)$ ist bei 94 Prozent. Der regelmäßige Puls bei 89. In der Belastung atmet Herr Müller durch den Mund. Er wird kurzatmig. Dabei sinkt die $\mathrm{SpO}_{2}$ und die Herzfrequenz steigt. Der Patient erholt sich innerhalb von 2-3 Minuten vollständig. Er hustet und würgt nach der Belastung, bis sich etwas Schleim löst.

$\rightarrow$ Palpation: Das Gewebe am Thorax ist prall und fest, die Muskulatur verspannt. Die thorakale Atembewegung auf der rechten Seite ist vermindert.

$\rightarrow$ Auskultation: Die Lungen sind gut belüftet, die Atemgeräusche eher verstärkt. Das ist ein Zeichen für einen erhöhten Widerstand in den größeren Atemwegen durch zähes Sekret.

$\rightarrow$ Belastungstest: Im „Sit-to-Stand-Test“ (Dauer: 1 Minute) schafft der Patient nur die Hälfte des Solls (18 Mal statt 36). Er gibt als limitierende Faktoren die Beinmuskulatur und die Atmung an.

$\rightarrow$ Fragebogen: Beim Ausfüllen des COPD Assessment Tests, CAT, erreicht der Patient einen Gesamtscore von 21 ( $\odot$ COPD ASSESSMENT TEST, CAT). Das spricht für eine dauerhaft eingeschränkte Lebensqualität und Belastbarkeit.

Physiotherapeutische Diagnose und Prognose/Risiken $\rightarrow$ Herr Müller kommt hilfesuchend in die Therapie. Er ist mit zähem Sekret und Hustenattacken konfrontiert. In der Folge schmerzen seine Rippen. Sein Übergewicht und sein Bewegungsmangel deuten auf eine Dekonditionierung hin. Außer der regelmäßigen Inhalation, die er gut ausführt, scheinen das Husten- und Sekretmanagement sowie seine Wahrnehmung der Krankheitssymptome ungenügend zu sein.

$\rightarrow$ Strukturhypothese 1: Die Rippenwirbelgelenke sind gereizt.

$\rightarrow$ Strukturhypothese 2: Das Übergewicht schränkt den Bewegungsradius ein.

$\rightarrow$ Funktionshypothese 1: Die Sekretelimination ist gestört. Die Konsistenz des Sekrets sowie die Technik sollten verbessert werden.

$\rightarrow$ Funktionshypothese 2: Die Schlafapnoe ist ein Zeichen der Obstruktion der oberen Atemwege.

Der Patient ist offen für konkrete Maßnahmen, die seine Lebensqualität verbessern. Er war früher Leistungssportler und kann sich auch vorstellen, ein Training zu absolvieren.

Behandlungsziele, -aufbau und Evaluationskriterien $\rightarrow$ Als Nahziele formuliert sein Physiotherapeut die Reduktion der Schmerzen
:

COPD Assessment Test (CAT)

\section{COPD-Fragebogen}

Der COPD Assessment Test (CAT) ist ein validierter Fragebogen zur Beurteilung und Verlaufskontrolle der COPD. Seine acht Fragen umfassen neben den unmittelbaren Symptomen von Seiten der Lunge auch allgemeine, den Alltag bestimmende Beschwerden. Seine Sensitivität ist ausreichend, um Veränderungen im Krankheitsbild zu erfassen.

Erreichen Patient*innen weniger als 10 Punkte, bedeutet das, dass die meisten Tage gut sind; die COPD hindert sie zwar an der einen oder anderen Aktivität, dauerhafte Beschwerden bestehen jedoch nicht. Sinnvoll ist die Wahrnehmung körperlicher Aktivität (Lungensport).

Bei über 30 Punkten sind die Lebensqualität und Belastbarkeit sehr stark und dauerhaft eingeschränkt; hier müssen alle verfügbaren Behandlungsmöglichkeiten (Beendigung des Tabakkonsums, Medikamente, Lungensport, Sauerstoffbehandlung) hinsichtlich eines möglichen Einsatzes geprüft werden [3].

Den Test findet man online unter: bit.ly/Test_CAT
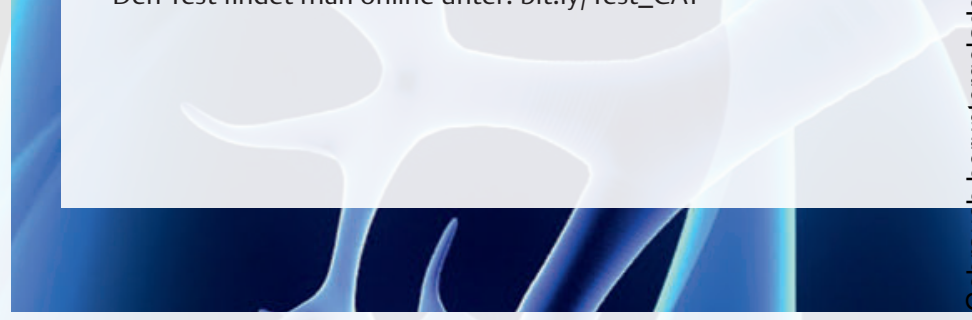

thorakal durch die Anlage eines elastischen Rippentapes sowie die Reduktion des Gewebewiderstands.

Mittelfristige Ziele sind eine verbesserte Atemwahrnehmung und Hustenkontrolle durch den Einsatz eines einfachen PEP-Systems (PEP: Positive Expiratory Pressure - exspiratorische Bremse) sowie die Atemwahrnehmung unter Belastung.

Langfristige Ziele sind die Teilnahme an einem Programm der pulmonalen Rehabilitation mit Patientenedukation und Trainingseinheiten sowie die apparative Versorgung während der Nacht. Folgende Evaluationskriterien legt er mit seinem Therapeuten fest:

$\rightarrow$ Schmerzen mittels Schmerzskala

$\rightarrow$ Anzahl der Hustenattacken/Mobilisierbarkeit des Sekrets

$\rightarrow$ Gesamtscore beim CAT

$\rightarrow$ Score beim „Sit-to-Stand-Test“

Ausschnitt aus einer Behandlungssequenz $\rightarrow$ Im Folgenden ist ein exemplarischer Ausschnitt aus einer Behandlungssequenz dargestellt. Der Einsatz eines einfachen Strohhalms - im Sinne einer PEPAtmung, die der Patient in einer atemerleichternden Stellung durchführt, hat die Atemwahrnehmung verbessert (॰ ABB. 2). Die Atmung wurde bewusster durchgeführt. Außerdem hat der Stroh- 


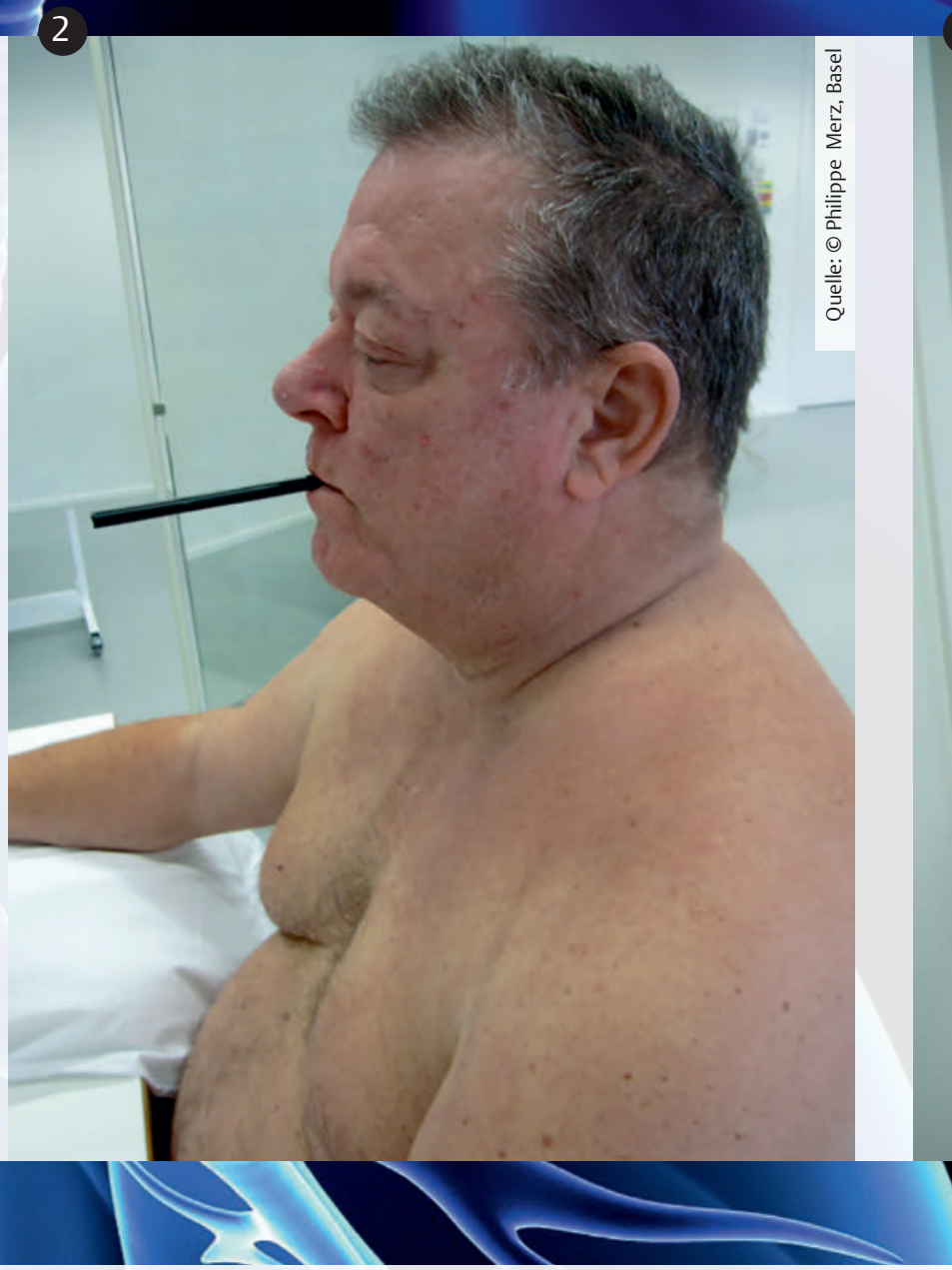

3

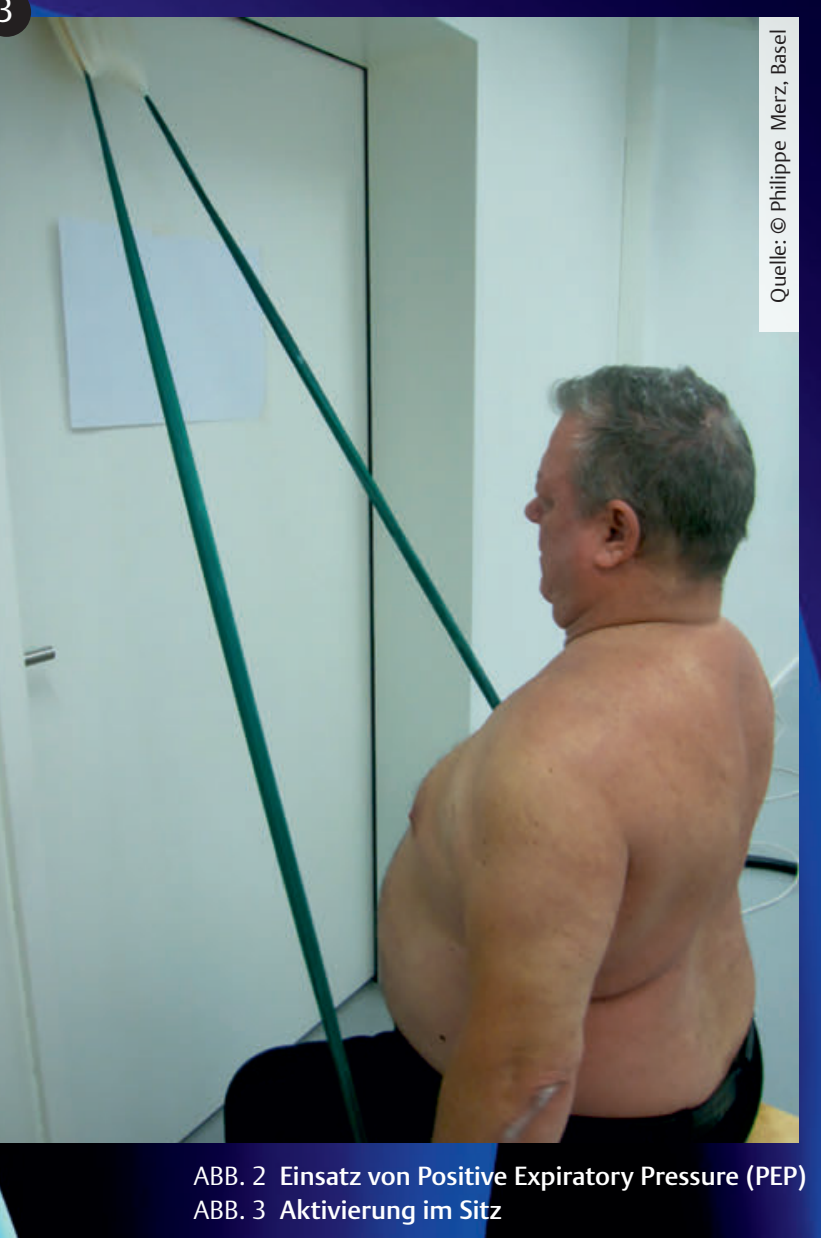

halm eine Atemvertiefung ermöglicht: Nach einer längeren Exspiration folgte eine vertiefte Einatmung. Und schließlich hat das Hilfsmittel eine kontrollierte und verlängerte Ausatmung ausgelöst. Das ist wichtig, da die verlängerte Atmung das Zentralisieren von Sekret unterstützt und das Abhusten erleichtert.

Der Patient war von dem einfachen Hilfsmittel „Strohhalm“ überzeugt und hat ihn sofort in seinem Alltag am Computer eingesetzt. In den folgenden Therapien hatte Herr Müller immer einen Strohhalm dabei. Die erfolgreiche Atemwahrnehmung in Ruhe konnte in eine kontrollierte Belastung im Sitz (○ ABB. 3) und später bei Belastung im Stand mit dem Theraband übernommen werden.

Für die manuelle Bearbeitung des Gewebes aus einer atemerleichternden Stellung im Sitz am Tisch sollte Herr Müller das Armgewicht auf Kissen abgeben. Das Lagern in Flexionsstellung der Schultergelenke brachte den Brustkorb in eine Einatmungsstellung. Die Hand des Therapeuten folgte den Atemexkursionen des Patienten und betonte danach die Exspiration mit einer Verschiebung des Gewebes zur Wirbelsäule hin. Herr Müller reagierte spontan mit einer vertieften Inspiration beim nächsten Atemzug. Im Anschluss ging es bei Herrn Müller darum, dass er Bewegung und Atmung miteinander koordiniert: Mit der Flexionsstellung in der Wirbel- säule kombinierte Herr Müller die Einatmung; mit dem extensorischen Aufrichten sollte er die Exspiration kombinieren.

Fazit $\rightarrow$ Menschen wie Herr Müller, die sehr motiviert in die Behandlung kommen und selbstverantwortlich üben, bringen gute Voraussetzungen für eine erfolgreiche Behandlung mit. Gibt man ihnen Hilfsmittel an die Hand, die sie einfach in den Alltag integrieren können, überzeugt das Patient*innen. Atemerleichternde Stellungen mit Gewichtabgabe und in einer Einatmungsstellung erleichtern das Atmen und optimieren den Einsatz der Hilfsmittel. Mithilfe einer Wahrnehmungsschulung befähigen Therapeut*innen die Patient*innen, in Ruhe und Belastung ihre Atmung zu kontrollieren.

Philippe Merz

$\mathbf{a}$ Literaturverzeichnis www.thieme-connect.de/products/physiopraxis > "Ausgabe 1/22“ 